\title{
Long non-coding RNA DUXAP8 elevates RCN2 expression and facilitates cell malignant behaviors and angiogenesis in cervical cancer via sponging miR-1297
}

Jihui Gu, Yi Liu, Ting Qi, Weiwei Qian, Dongdong Hu and Wen Feng*

\begin{abstract}
Background: Cervical cancer (CC) endangers women's health in the world range. Accumulating studies have revealed the crucial regulatory role of long non-coding RNAs (IncRNAs) in multiple malignancies, including CC. Our study aimed to explore the role of IncRNA double homeobox A pseudogene 8 (DUXAP8) in cervical carcinogenesis.

Methods: Gene expressions in CC were assessed by RT-qPCR. Function experiments and tube formation assays were performed to evaluate the role of DUXAP8 in CC cells. Subcellular fractionation and FISH assays were conducted to determine the subcellular location of DUXAP8. Luciferase reporter, RNA pull down and RIP assays were conducted to investigate the mechanism of DUXAP8.

Results: DUXAP8 was notably upregulated in CC cells. Downregulation of DUXAP8 repressed cell malignant behaviors and angiogenesis in CC. Mechanically, DUXAP8 boosted the expression of reticulocalbin-2 (RCN2) through relieving the binding of miR-1297 to RCN2 3'-UTR. Moreover, miR-1297 inhibition and RCN2 overexpression could counteract the inhibitory effects of DUXAP8 knockdown on the malignant phenotypes of CC cells. Besides, enhanced RCN2 expression restored the tumor growth in vivo that was inhibited by DUXAP8 repression.

Conclusions: DUXAP8 promotes malignant behaviors in CC cells via regulating miR-1297/RCN2 axis.

Keywords: Cervical cancer, DUXAP8, miR-1297, RCN2
\end{abstract}

\footnotetext{
*Correspondence: 19116701@qq.com

Department of Gynecology, the First People's Hospital of Lianyungang, No.6

Zhenhua East Road, Jiangsu 222000 Lianyungang, China
}

(c) The Author(s). 2021 Open Access This article is licensed under a Creative Commons Attribution 4.0 International License, which permits use, sharing, adaptation, distribution and reproduction in any medium or format, as long as you give appropriate credit to the original author(s) and the source, provide a link to the Creative Commons licence, and indicate if changes were made. The images or other third party material in this article are included in the article's Creative Commons licence, unless indicated otherwise in a credit line to the material. If material is not included in the article's Creative Commons licence and your intended use is not permitted by statutory regulation or exceeds the permitted use, you will need to obtain permission directly from the copyright holder. To view a copy of this licence, visit http://creativecommons.org/licenses/by/4.0/ The Creative Commons Public Domain Dedication waiver (http://creativecommons.org/publicdomain/zero/1.0/) applies to the data made available in this article, unless otherwise stated in a credit line to the data. 


\section{Graphical Abstract}

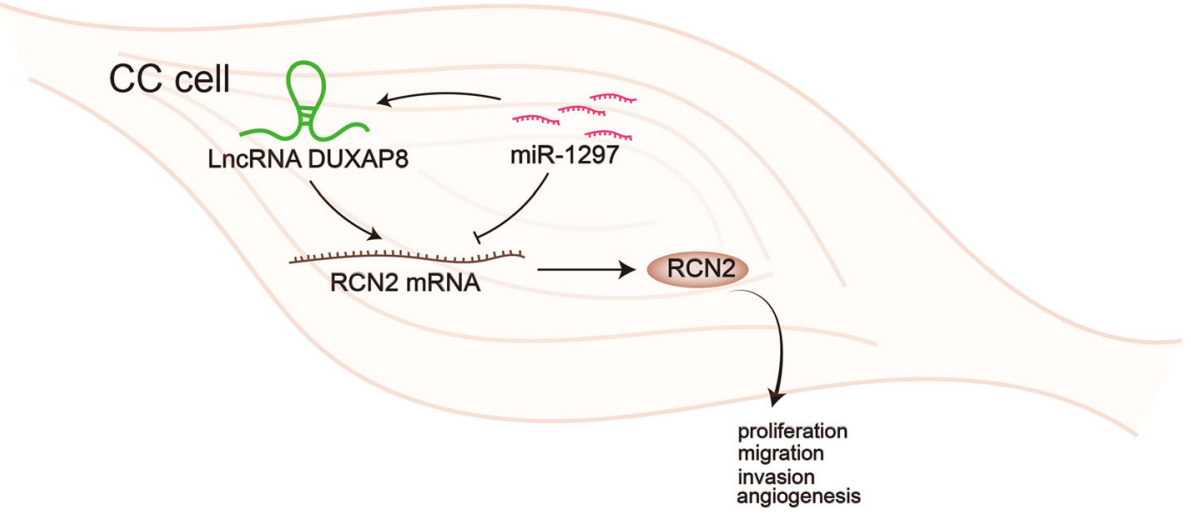

\section{Background}

Cervical cancer (CC) remains one of the most common malignancies that undermines the health of females and a major health challenge in developing countries $[1,2]$. Over the past decade, surgery operation, radiation oncology and chemotherapy have been developed to combat CC, yet the recurrence rate for patients remains $35 \%$ [3]. In 2021, the estimated number of new cases of CC is 14,480 and the estimated death number of CC is 4290 in the United States [4]. Numerous reports have focused on gene therapy and explored the regulations between genes in various types of cancers. Herein, we aimed to elucidate the mechanism behind the carcinogenesis of CC.

Long non-coding RNAs (lncRNAs) are a group of non-coding RNAs (ncRNAs) over 200 nucleotides [5]. The dysregulation of lncRNAs in cellular processes has been discovered in an array of cancers, indicating the potential application of lncRNAs as diagnosis biomarkers and therapeutic targets for cancers [6] Meanwhile, findings on the molecular mechanisms and pathways of lncRNAs can be translated into clinical treatment for patients [7]. A great number of lncRNAs have been reported to play pivotal roles in a series of cellular biological processes, such as cell proliferation, differentiation, apoptosis, migration and epithelial mesenchymal transition (EMT) [8, 9]. For instance, DUXAP8 knockdown suppresses cell proliferation and facilitates cell apoptosis in pancreatic cancer [10]. Moreover, lncRNAs have been discovered to function as a class of competing endogenous RNAs (ceRNAs) to posttranscriptionally regulate gene expressions. Under ceRNA networks, lncRNAs could restore the expression of specific messenger RNAs (mRNAs) via sponging miRNAs through physical interaction [11]. For example, DUXAP8 promotes the progression of hepatocellular carcinoma by sponging miR-422a and enhancing PDK2 expression [12]. DUXAP8 serves as ceRNA for miR-577 to facilitate the invasion and migration of colorectal cancer cells via regulating RAB14 [13]. The similar mechanism of lncRNAs in CC has also been uncovered. For instance, silencing LINC01305 inhibited the PI3K/Akt signaling pathway in CC via targeting TNXB [14]. CAR10 facilitates CC development by sponging miR125b-5p and upregulating PDPK1. However, the performance of DUXAP8 in CC remains unexplored.

MiRNAs represent another kind of ncRNAs, which possess approximately 18-22 nucleotides in length [15]. To date, miRNAs have been extensively reported to participate in the pathogenesis of various cancers [16]. More importantly, previous studies have highlighted the biological function of miRNAs in CC [17].

As a member of reticulocalbin (RCN) family, reticulocalbin-2 (RCN2) was revealed to exert oncogenic effects in several human malignancies. As an example, RCN2 was unveiled to be up-regulated in hepatocellular carcinoma $(\mathrm{HCC})$ and its expression was positively correlated with tumor size in HCC patients [18]. However, the relationship between RCN2 and DUXAP8 has never been investigated in CC.

In this study, we aimed to investigate whether and how DUXAP8 regulates CC progression. The expression pattern of DUXAP8 was firstly determined in CC cells. Functions of DUXAP8 in CC progression were also detected. Importantly, we focused on the downstream molecular mechanism of DUXAP8 in CC. Collectively, we probed into the functions of DUXAP8 via specific miRNA/mRNA axis in CC.

\section{Methods}

\section{Cell culture}

Human CC cell lines (C4-1, caSki, HeLa and SiHa) and normal cervical cell line (Ect1/E6E7) were all procured from Shanghai Institute of Cell Biology (Shanghai, 
China). All cells were propagated in RPMI-1640 with $1 \%$ antibiotics and $10 \%$ FBS (Gibco, Grand Island, NY) under $5 \% \mathrm{CO}_{2}$ at $37^{\circ} \mathrm{C}$.

\section{Total RNA extraction and real-time quantitative PCR (RT- qPCR)}

Total RNAs were extracted from caSki and HeLa cell samples as instructed by the protocol of TRIzol reagent (Invitrogen, Carlsbad, CA). After that, RNA samples were subjected to PrimeScript ${ }^{\mathrm{Tm}}$ RT Master Mix (TaKaRa, Shiga, Japan) for cDNA synthesis. SYBR Green PCR Kit (TaKaRa) was employed to perform qPCR, with GAPDH and U6 as the endogenous controls. Relative RNA expression was processed by $2^{-\Delta \Delta C t}$ method.

\section{Plasmid transfection}

Silencing of DUXAP8 and overexpression of RCN2 in caSki and HeLa cell samples were severally achieved by transfection with the duplex shRNAs (Genepharma, Shanghai, China) against DUXAP8 and pcDNA3.1/ RCN2. The nonspecific shRNAs and empty pcDNA3.1 vector (Invitrogen) served as the negative control (NC). Besides, miR-1297 mimics/inhibitor and NC mimics/inhibitor were also procured from Genepharma. Cell transfection was performed for $48 \mathrm{~h}$ with Lipofectamine 2000 (Invitrogen).

\section{Colony formation assay}

$\mathrm{CC}$ cells in the 6 -well plates were seeded at $1 \times 10^{3}$ cells/ $100 \mu \mathrm{L}$ for 14 days of incubation at $37^{\circ} \mathrm{C}$ with $5 \% \mathrm{CO}_{2} .1$ $\mathrm{mL} 0.1 \%$ crystal violet solution was added to each well prior to counting colonies.

\section{EdU incorporation assay}

CC cells were planted after transfection into 96-well plates with $8 \times 10^{3}$ cells/well and then processed with the Cell-light ${ }^{\mathrm{Tm}}$ EdU ApolloR567 in Vitro Imaging Kit (RiboBio, Guangzhou, China). Following fixation and permeabilization, samples were imaged by fluorescent microscope (Olympus Corp., Tokyo, Japan) after DAPI staining for cell nuclei.

\section{Annexin V staining assay}

Transfected CC cell samples were harvested, rinsed in pre-chilled phosphate buffered saline (PBS), and then exposed to Annexin V FITC/PI detection kit (Invitrogen) as instructed by supplier for $15 \mathrm{~min}$ at $4^{\circ} \mathrm{C}$. Finally, cell apoptosis rate was evaluated by analyzing Annexin V/PIstained cells via flow cytometer (Beckman Coulter, Inc., Brea, CA).

\section{Transwell assay}

Transwell assays were implemented using the transwell chamber (Corning Co, Corning, NY) with Matrigel (for invasion assay) or without Matrigel (for migration assay) as per the protocol. $5 \times 10^{4}$ cell samples were added into the upper chamber, with complete medium added into the lower chamber. Invading or migrating cells were fixed in $4 \%$ paraformaldehyde after $24 \mathrm{~h}$, and then dyed with crystal violet solution. Stained cell samples were photographed under microscope and quantified.

\section{Tube formation assay}

$2.5 \times 10^{4}$ human vascular endothelial cells (HUVECs) cultured in the conditional medium (CM) of caSki or HeLa cells were grown in 96-well plates with Matrigel at $37^{\circ} \mathrm{C}$ for $6 \mathrm{~h}$. The branches, representing the degree of angiogenesis in vitro, were observed and the number was quantitated under a light microscope (Nikon, Tokyo, Japan).

\section{Subcellular fractionation assay}

Based on the protocol, the nuclear and cytoplasmic fractions were isolated from cell samples using PARIS ${ }^{\mathrm{ma}} \mathrm{Kit}$ (Invitrogen). After treating with the cell fractionation and cell disruption buffer in sequence, DUXAP8 expression level in different fractions was determined by RTqPCR, with GAPDH and U6 as indicated controls.

\section{RNA FISH analysis}

For RNA FISH analysis, CC cell samples were fixed and air-dried for cultivation with DUXAP8 FISH probe (RiboBio) in the hybridization buffer. Hoechst solution was then added for the detection of cell nuclei, followed by the cells visualized with fluorescent microscope.

\section{Luciferase reporter assays}

Recombinant luciferase reporter vectors DUXAP8 WT/ Mut or RCN2 WT/Mut were acquired by inserting DUXAP8 or RCN2 fragments with the wild-type or mutated miR-1297 binding sites into the downstream of reporter vector pmirGLO (Promega, Madison, WI). Then the recombinant vectors were subjected to cotransfection with miR-1297 mimics or NC mimics for 48 h. After that, Luciferase Reporter Assay System (Promega) was employed for detecting the luciferase activity.

\section{RNA pull down assay}

The wild type or mutant miR-1297 sequences covering DUXAP8 binding sites were synthesized and tagged with biotin into Biotin miR-1297 WT/Mut. The extracts from CC cell samples were mixed with the biotinylated RNA probes and streptavidin beads. The RNA in final pulldowns was assayed through RT-qPCR.

RNA binding protein immunoprecipitation (RIP)

RIP assay was conducted by use of EZ-Magna RIP RNA Binding Protein Immunoprecipitation Kit (Millipore, 
Bedford, MA) as required by supplier. Cultured cell samples were lysed and then mixed with the beads-bound human anti-Ago2 antibody or normal control anti-IgG antibody (Millipore). After RNA purification, RT-qPCR analysis was conducted to verify the presence of indicated RNAs.

\section{In vivo xenograft experiments}

Nine six-week-old BALB/c nude mice were acquired from Vital River Laboratories (Beijing, China). Xenograft tumor models were established via subcutaneously injecting caSki cells transfected with sh-NC, shDUXAP8\#1 or sh-DUXAP8\#1 + pcDNA3.1/RCN2 into above mice (randomly divided into three groups). The volume of neoplasms was monitored every 4 days. Four weeks later, mice were sacrificed and the weight of each tumor was measured. The animal study was approved by the Ethics Committee of the First People's Hospital of Lianyungang.

\section{Statistical analysis}

Data from independent bio-triplications were expressed as the mean $\pm \mathrm{SD}$. Differences between the different groups were processed through $t$ test or one-way analysis of variance applying the PRISM 7.0 (GraphPad, San Diego, CA). Threshold of significance was set as $p$ value $<0.05$.

\section{Results}

DUXAP8 is aberrantly highly expressed in CC and silencing DUXAP8 suppresses the malignant behaviors of CC cells

RT-qPCR analysis was performed in CC cell lines and normal cervical epithelial cell line Ect1/E6E7 to explore the expression level of DUXAP8. Results manifested that DUXAP8 expression was markedly increased in CC cell lines, especially in caSki and HeLa cells (Fig. 1A), which suggested that DUXAP8 might be implicated in the progression of CC. To determine the role of DUXAP8 in $\mathrm{CC}$, we adopted loss-of-function assays after ensuring the inhibitory efficiency of sh-DUXAP8\#1/2 in caSki and HeLa cells (Fig. 1B). Colony formation and EdU assays indicated that silencing DUXAP8 evidently suppressed the proliferation of caSki and HeLa cells (Fig. 1C, D). In addition, flow cytometry analyzed that the rate of apoptotic cells was increased by silencing DUXAP8 (Fig. 1E), implying the promoting effect of DUXAP8 knockdown on CC cell apoptosis. More importantly, we found that the absence of DUXAP8 could impair the migration and invasion capacities of caSki and HeLa cells (Fig. 1F, G). Since angiogenesis is crucial for tumor growth and metastasis, we performed tube formation assay in HUVECs incubated with the conditional medium (CM) of caSki and HeLa cells. As a result, loss of DUXAP8 reduced the number of tube branches (Fig. $1 \mathrm{H})$, indicating the repressive impact of downregulated DUXAP8 on angiogenesis. In sum, DUXAP8, upregulated in CC, strengthens the malignant phenotypes of CC cells.

\section{MiR-1297 binds to DUXAP8 and is negatively mediated by DUXAP8}

The ceRNA network is an important posttranscriptional regulatory mechanism of cytoplasmic IncRNAs. From the outcomes of subcellular fractionation and FISH assays, we found that DUXAP8 mainly located in the cytoplasm of caSki and HeLa cells (Fig. 2A, B). This phenomenon indicated the possible ceRNA role of DUXAP8 in CC. By the prediction on starBase (http:// starbase.sysu.edu.cn/) database, 10 miRNAs with potential binding capacity to DUXAP8 were detected (Clip data: medium stringency $\geq 2$, Class: $>7$ mer-m8). After performing RT-qPCR analysis, only miR-1297 was found to be conspicuously up-regulated in response to DUXAP8 deficiency (Fig. 2C). Then, we browsed starBase and acquired the putative binding sites between DUXAP8 and miR-1297 (Fig. 2D). Subsequently, miR1297 was found to be downregulated in CC cells compared with normal ones (Fig. 2E). According to the results of luciferase reporter assays, we found that miR1297 mimics notably attenuated the luciferase activity of DUXAP8-WT in caSki and HeLa cells, while presented no effects on that of DUXAP8-Mut (Fig. 2F). Moreover, the data of RNA pull down assay manifested that DUXAP8 was enriched only by Biotin miR-1297 WT (Fig. 2G). These results indicated that DUXAP8 directly interacted with miR-1297 and negatively regulated miR1297 expression in CC cells. We utilized miR-1297 inhibitor to down-regulate the expression of miR-1297 in HeLa cells (Fig. 2H). After conducting a series of rescue experiments, we verified that miR-1297 inhibition distinctively abrogated the cellular effects mediated by silencing DUXAP8 on different behaviors, including cell proliferation (Fig. 2I J), apoptosis (Fig. 2K), migration (Fig. 2L), invasion (Fig. 2M) and angiogenesis (Fig. 2N). To be concluded, miR-1297 was the downstream molecule of DUXAP8 in regulating CC development.

\section{RCN2 is the potential downstream target of miR-1297}

Numerous studies discovered that IncRNAs could serve as ceRNAs to affect the expression of genes targeted by the shared miRNAs, leading to changes in corresponding biological function in cancer. We used starBase database to search potential targets of miR-1297. As a result, four candidates (RCN2, CHORDC1, TMEM184B and ARPC3) were predicted to interact with miR-1297 under following circumstances (CLIP Data: high stringency $\geq 3$; 


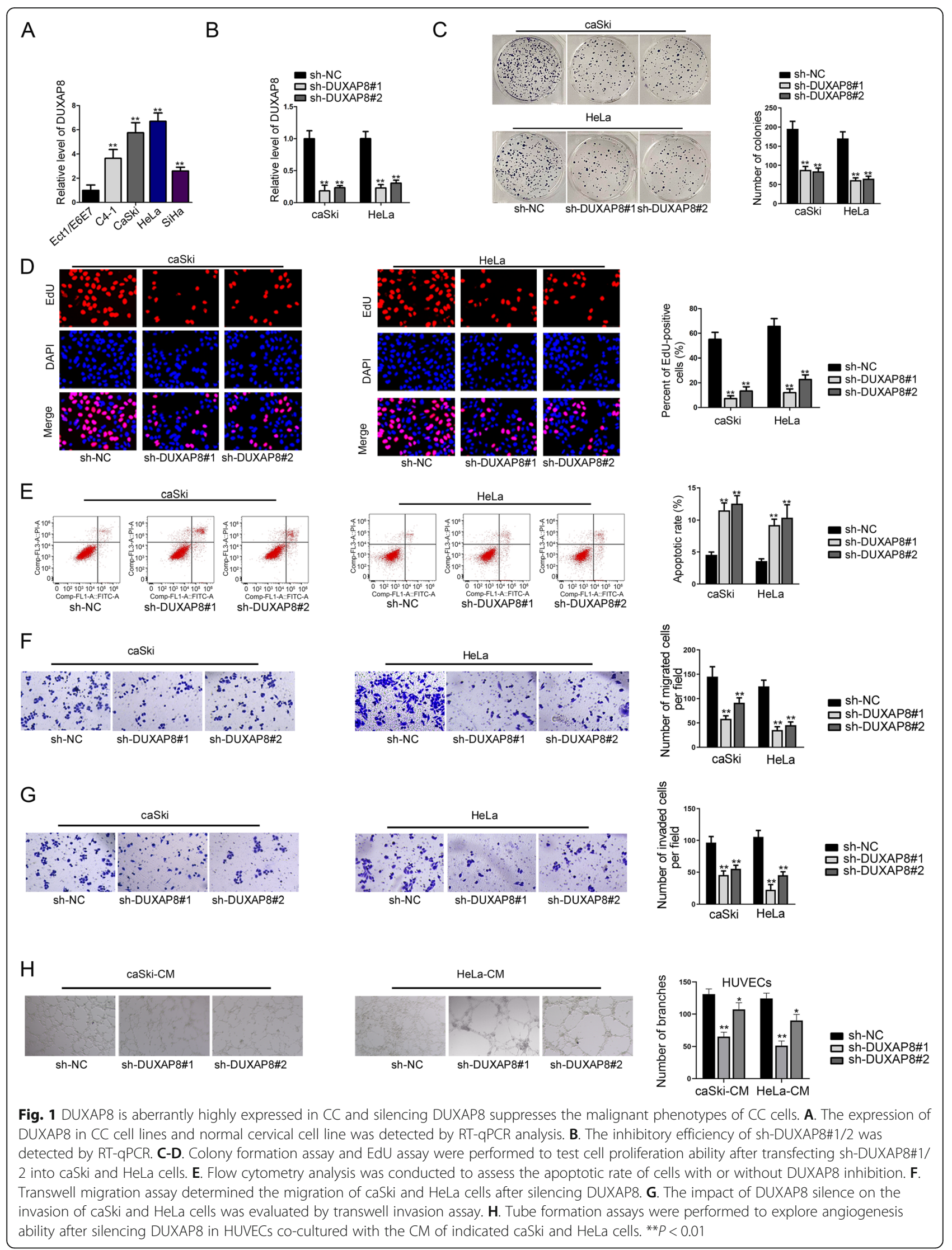




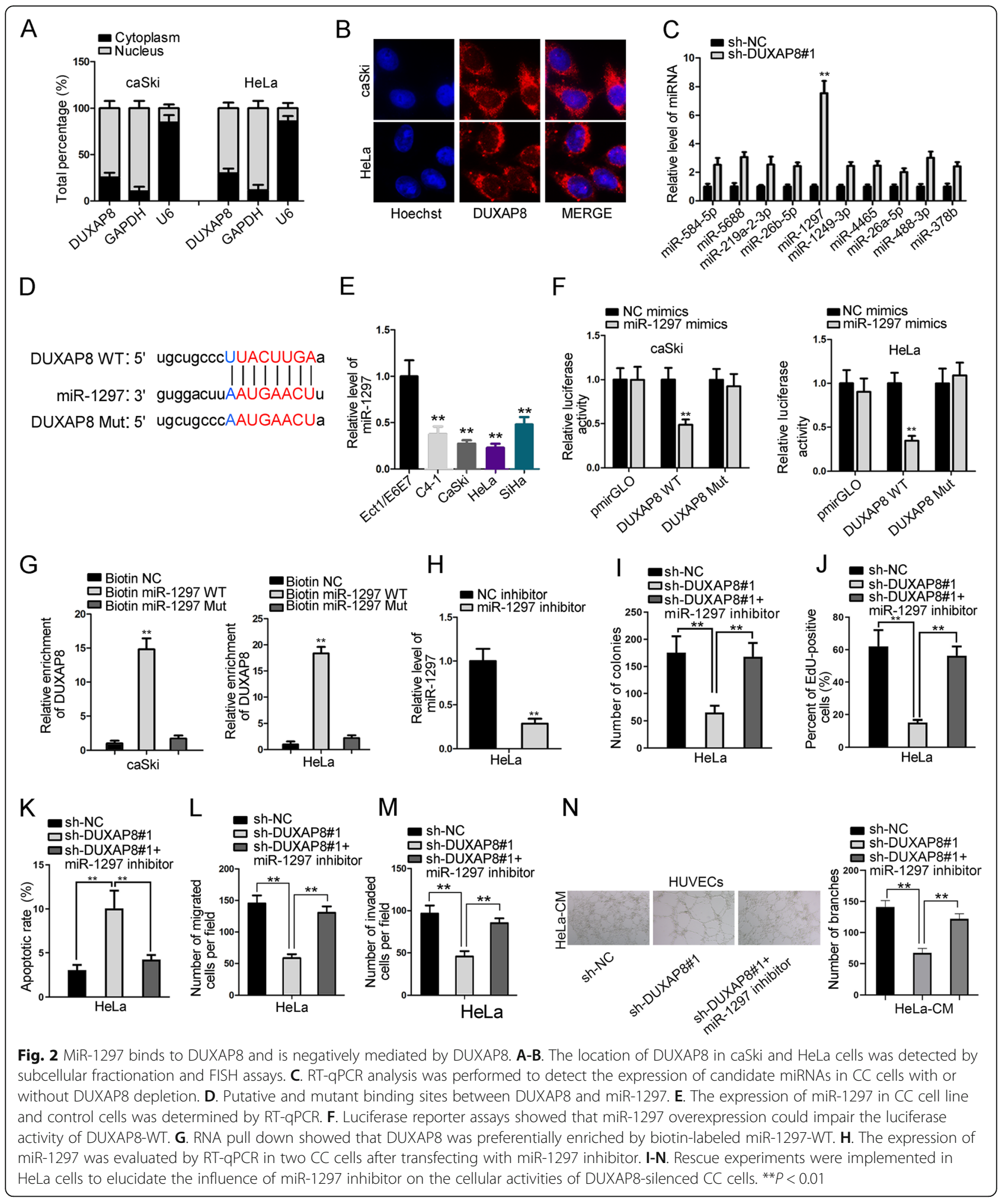

Degradome Data: high stringency $\geq 3$, Program Number: 5, Predicted Program: microT, miRanda, miRmap, PITA). Later, RT-qPCR results showed that RCN2 expression was depleted most significantly after HeLa cells was transfected with miR-1297 mimics (Fig. 3A). Also, we observed a significant up-regulation of RCN2 in CC cell lines (Fig. 3B). The putative binding site of miR1297 in the 3' UTR sequence of RCN2 was predicted by 


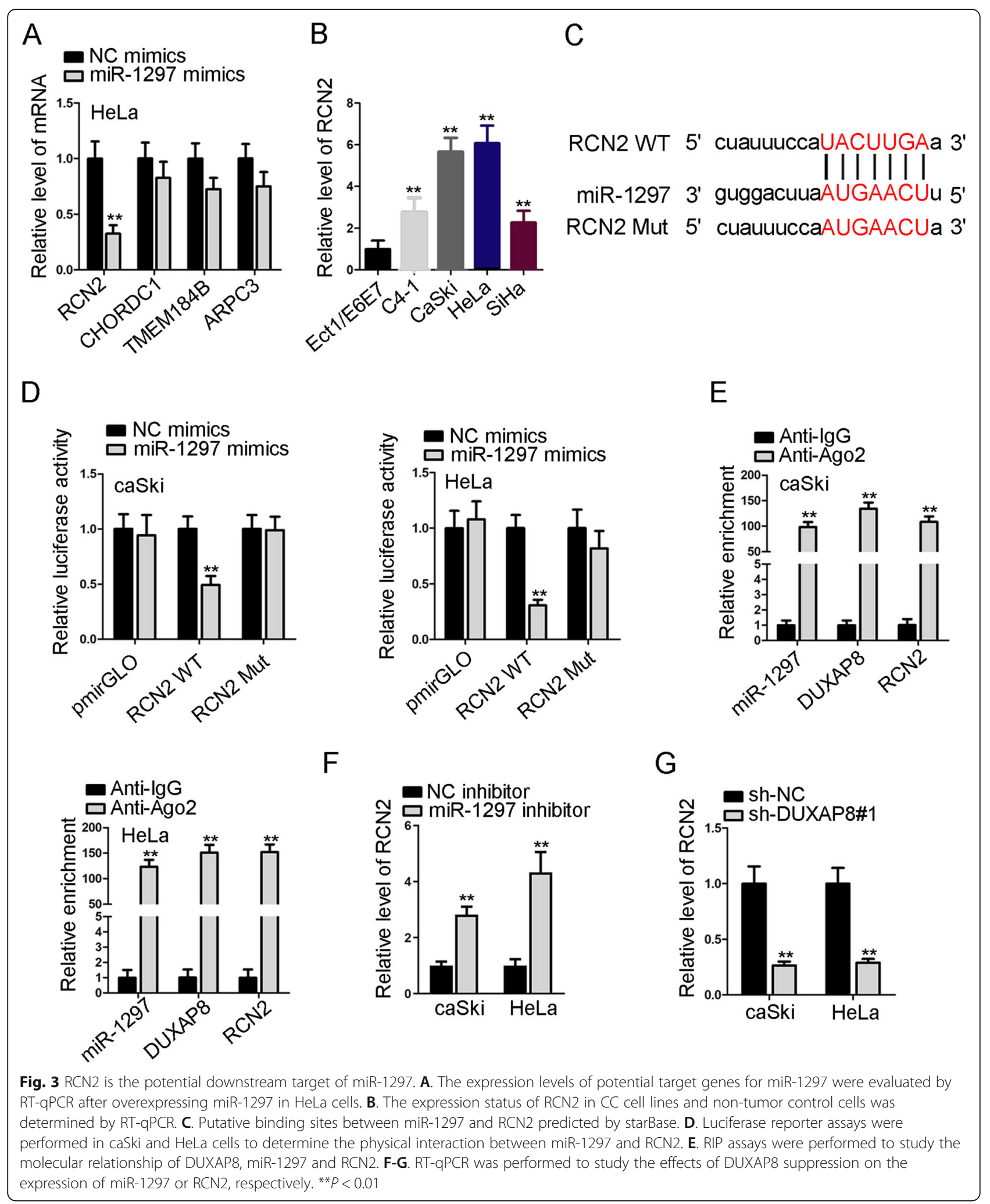

starBase database (Fig. 3C). Data of luciferase reporter assays demonstrated that miR-1297 overexpression could significantly impair the luciferase activity of
RCN2-WT in caSki and HeLa cells, whereas no changes in the luciferase activity was observed in RCN2-Mut group (Fig. 3D). Additionally, RIP assay was used to 


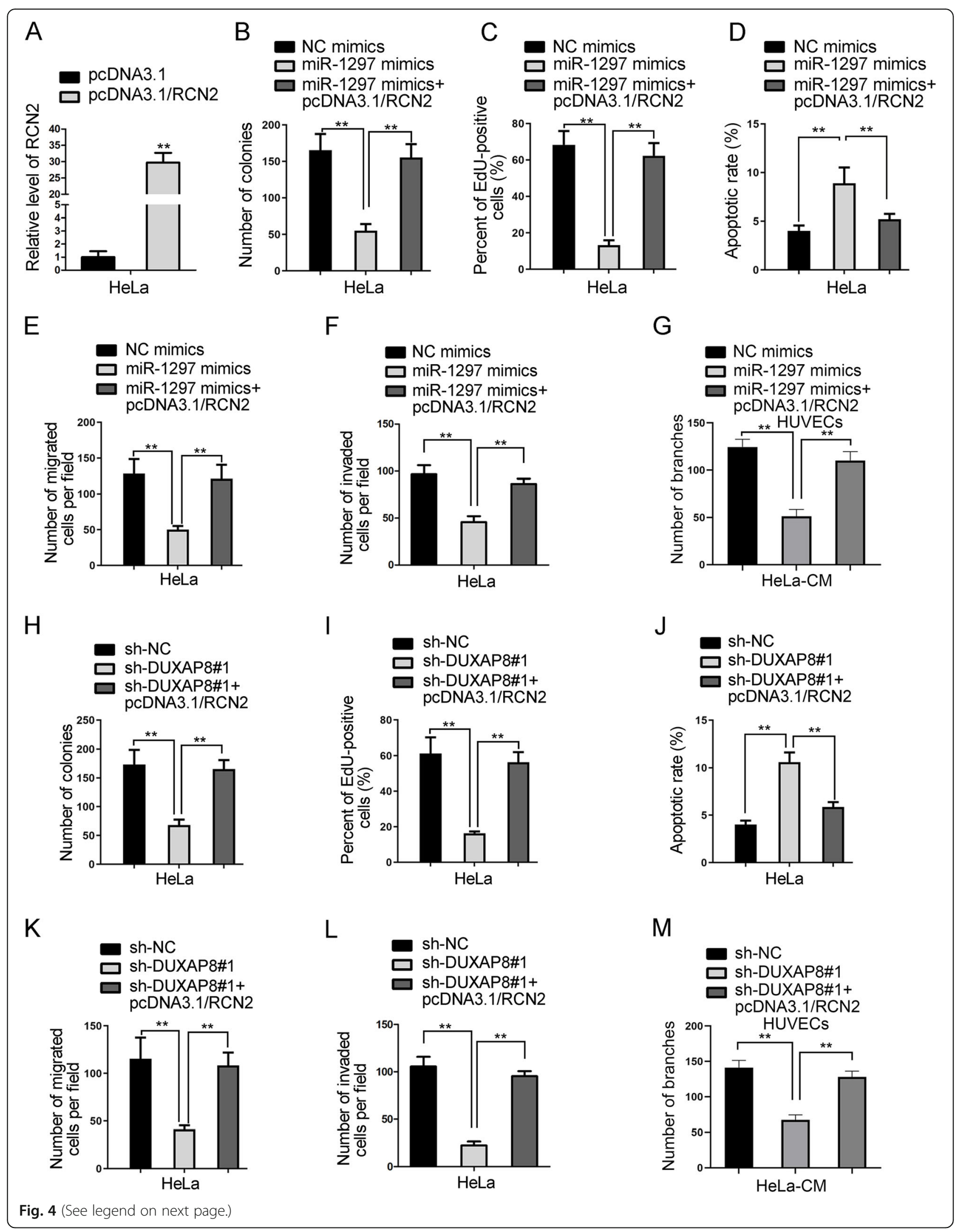


(See figure on previous page.)

Fig. 4 LnCRNA DUXAP8 regulates CC cellular behaviors via up-regulating RCN2 by miR-1297. A. The expression of RCN2 in HeLa cells after transfecting with pcDNA3.1/RCN2 was detected by RT-qPCR. B-C. Colony formation and EdU assays were conducted to explore the proliferation of HeLa cells transfected with NC mimics, miR-1297 mimics or miR-1297 mimics + pcDNA3.1/RCN2. D. Flow cytometry analysis was performed to assess the rate of apoptotic cells under different conditions. E-G. Transwell and tube formation assays were conducted to determine cell migration, invasion and angiogenesis under diverse contexts. $\mathbf{H}-\mathbf{M}$. Rescue functional experiments were performed among sh-NC, sh-DUXAP8\#1 and sh-DUXAP8\#1 + pCDNA3.1/RCN2 groups to explore the effects of RCN2 overexpression on DUXAP8 inhibition-mediated function in HeLa cells. ${ }^{* *} P<0.01$

confirm the potential interaction between miR-1297 and DUXAP8, as well as the binding between miR-1297 and RCN2. It was showed that miR-1297, DUXAP8 and RCN2 were all markedly enriched by antibody targeting Ago2 compared with IgG control group (Fig. 3E). Further, we observed that the expression of miR-1297 was up-regulated by DUXAP8 silence, while that of RCN2 was depleted under DUXAP8 inhibition (Fig. 3F, G). These results supported that DUXAP8 works as a ceRNA of RCN2 in CC by sponging miR-1297.

\section{DUXAP8 regulates the behaviors of CC cells via up- regulating RCN2 by binding to miR-1297}

To certify whether DUXAP8 regulated $C C$ via serving as a ceRNA, we conducted rescue experiments in HeLa cells. We firstly overexpressed RCN2 in HeLa cells by transfecting with pcDNA3.1/RCN2 (Fig. 4A). It was proved that the proliferation ability of CC cells was suppressed by miR-1297 up-regulation, but restored by RCN2 overexpression (Fig. 4B C). Meanwhile, the apoptotic ability was strengthened by enhanced miR-1297, but weakened by RCN2 overexpression (Fig. 4D). Moreover, the results of transwell assays showed that the inhibitory impacts of miR-1297 elevation on the migration and invasion of HeLa cells were counteracted by RCN2 overexpression (Fig. 4E F). The angiogenic ability restrained by miR-1297 up-regulation was recovered under RCN2 overexpression (Fig. 4G). These findings indicated that the anti-cancer biological function of miR-1297 was mediated by targeting RCN2. Next, we intended to explore whether RCN2 was required for DUXAP8mediated $\mathrm{CC}$ cell function. Based on the results of rescue experiments, we deduced that RCN2 up-regulation could reverse the proliferation-inhibitory impact of silenced DUXAP8 in HeLa cells (Fig. 4H, I). Besides, RCN2 up-regulation could counteract the apoptosispromoting effect exerted by depleted DUXAP8 (Fig. 4J). Also, the mitigating effects of inhibited DUXAP8 on HeLa cell migration and invasion were abolished in face of RCN2 up-regulation (Fig. 4K, L). The observation from tube formation assay further validated that RCN2 enhancement could recover the suppression of silenced DUXAP8 on angiogenesis (Fig. 4M). Altogether, DUXAP8 aggravates the malignant behaviors of CC cells through boosting RCN2 that is targeted by miR-1297.

\section{DUXAP8 contributes to in vivo CC tumorigenesis in an RCN2-mediated manner}

To further testify the importance of DUXAP8/RCN2 axis in $\mathrm{CC}$ tumorigenesis, we implemented in vivo xenograft experiments. According to the final view of xenografts and the corresponding growth curves, we discovered that the growth of tumors derived from DUXAP8-silenced CC cells was evidently obstructed compared to those from the control group, while such obstruction on tumor growth was markedly offset under RCN2 overexpression (Fig. 5A-B). Consistently, changes in tumor weight were similar to that in tumor growth rate in these three groups (Fig. 5C). Moreover, we found that the expression of DUXAP8 was lowered while that of miR-1297 augmented in xenografts from DUXAP8inhibited cells, while further overexpression of RCN2 had no impacts on these two aspects. Nonetheless, RCN2 level was also declined in tumors with suppressed DUXAP8, which was then recovered upon RCN2 upregulation (Fig. 5D). Besides, we found on TCGA dataset that DUXAP8 is positively correlated with RCN2 in CC tissues (Fig. 5E). Based on these data, we concluded that DUXAP8 facilitated the tumorigenesis of CC by regulating RCN2 that was targeted by miR-1297.

\section{Discussion}

$\mathrm{CC}$ is one of the most common diagnosed cancers in female. In gynecologic tumors, the incidence of $\mathrm{CC}$ is only preceded by breast cancer [19]. Primary therapies for CC patients includes surgery or a concurrent chemoradiotherapy treatment including cisplatin-based chemotherapy with brachytherapy and external beam radiotherapy [20]. However, the efficacy of cisplatin was compromised due to drug resistance feature of $\mathrm{CC}$ cells in treating advanced or recurrent CC [21]. Recently, the anti-cancer research on CC has switched to molecular level [20] while as a novel approach, gene therapy have brought hope to notably improve the survival rate of patients [22]. The underlying regulatory mechanism behind the pathogenesis of $\mathrm{CC}$ is worthwhile for anti-cancer research. Extensive studies have revealed that lncRNAs could affect various biological actions, such as transcriptional control, posttranscriptional regulation and the suppression of nuclear transport [23]. Moreover, the important regulatory role of lncRNAs in $\mathrm{CC}$ has been 


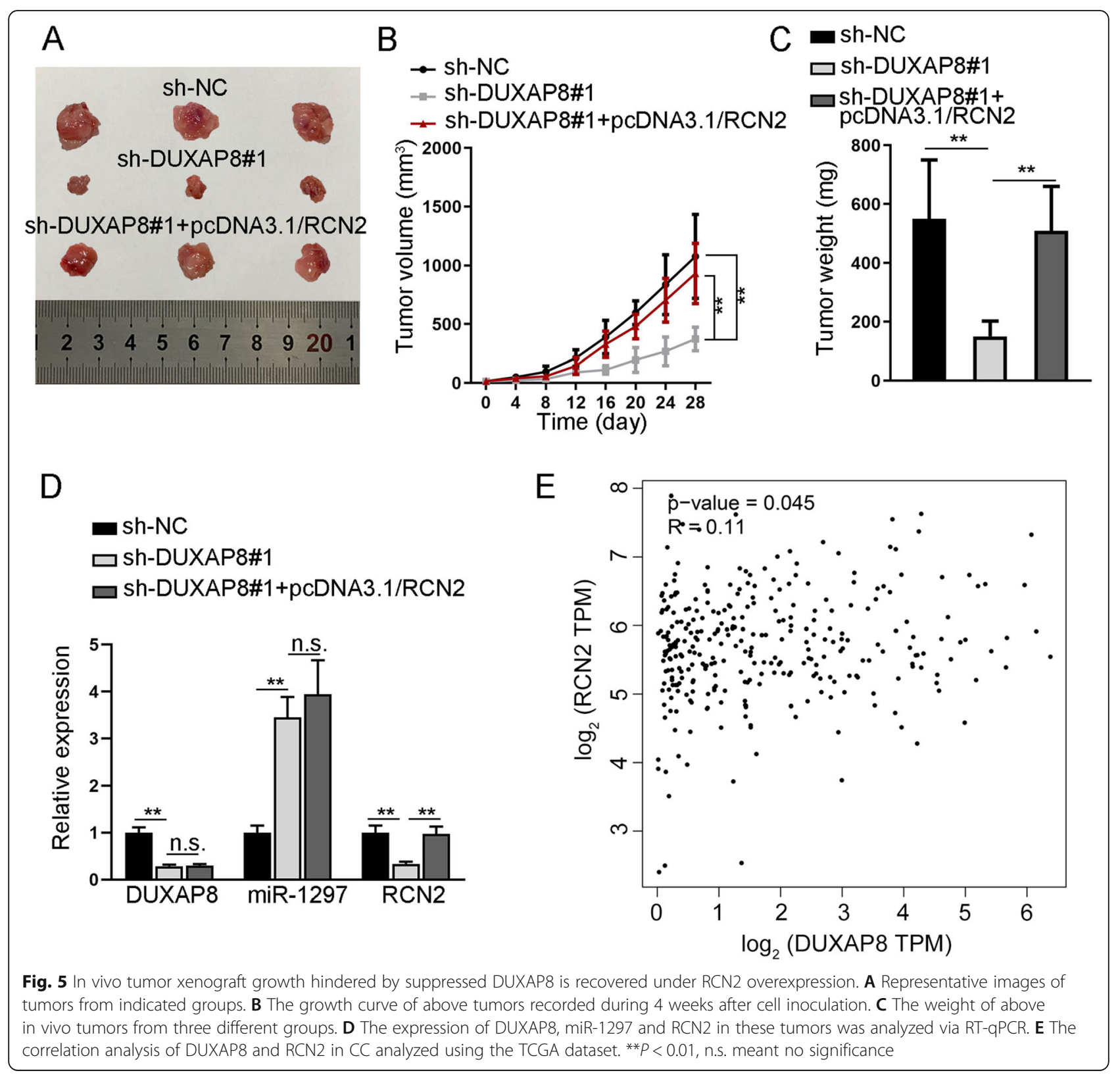

disclosed. DUXAP8, as a newly-found lncRNA, has been unveiled to possess oncogenic potential in gastric cancer [24]. Herein, we initially found the aberrant up-regulation of DUXAP8 in CC. Studies have shown that DUXAP8 facilitated cell growth in renal cell carcinoma and served as a promising biomarker in this disease [25]. Loss-of-function assays manifested that DUXAP8 silence suppressed cell proliferation, migration, and invasion in CC. Moreover, silencing DUXAP8 significantly weaken the angiogenesis ability in HUVECs incubated with the CM of CC cells. These observations revealed that DUXAP8 acted as an oncogene in CC, in line with the findings in HCC [26].

Increasing lncRNAs have been found to be involved in the prevalent ceRNA networks, where they could compete with the upstream mediators of mRNAs, miRNAs, for the 3'UTR region of mRNAs, consequently regulating such mRNAs [27, 28]. MiRNAs are a group of endogenous small ncRNAs that can modulate target gene expression at post-transcriptional level and capable of interaction with mRNA-coding genes [29]. Meanwhile, miRNA and IncRNA are key non-coding RNAs because of their negative feedback interaction and regulation on various biological processes by targeting diverse molecular pathways [30]. In our research, miR1297 was identified as a molecular downstream gene of DUXAP8 from bioinformatics analysis and mechanical experimental exploration. It has been uncovered that miR-1297 exerts inhibitory effects on the 
development and progression of multiple cancers, such as oral squamous cell carcinoma, [31] pancreatic cancer [32] and gastric cancer [33]. Similarly, miR1297 has been reported to function as a tumor suppressor to curb cell proliferation and facilitate cell apoptosis in CC [34]. Herein, based on rescue assays conducted in HeLa cells who have a high heterogeneity [35], we discovered that DUXAP8 exerted the oncogenic property in $\mathrm{CC}$ via physically binding to miR-1297 and negatively regulating its expression.

Reticulocalbin-2 (RCN2) was validated to elicit an oncogenic function in colorectal cancer [36]. In this study, it was identified as the target gene of miR-1297 by bioinformatics prediction and its level was significantly depleted by miR-1297 overexpression in CC cells. Mechanically, RCN2 overexpression markedly reversed the anti-tumor effects induced by miR-1297 overexpression and DUXAP8 knockdown on CC cells, indicating that DUXAP8 regulated the progression of $\mathrm{CC}$ by targeting miR-1297/RCN2 axis. Moreover, data of in vivo experiments further indicated that DUXAP8 contributed to the tumorigenesis of $\mathrm{CC}$ via modulating $\mathrm{RCN} 2$ that was targeted by miR-1297.

\section{Conclusions}

In sum, we initially found that DUXAP8/miR-1297/ RCN2 ceRNA axis elicited important impacts on cell proliferation, migration and invasion and angiogenesis in CC. In other words, DUXAP8 facilitated the malignant progression of CC cells via competitively binding with miR-1297 and upregulating RCN2 both in vitro and in vivo. This result provided new viewpoints into the potential therapeutic effects of DUXAP8 in CC, indicating that DUXAP8 may work as a practical biomarker and a treatment target for $\mathrm{CC}$ in the future. Therefore, our findings may shed new lights on the identification of potential novel anti-tumor targets for CC treatment.

\section{Abbreviations \\ CC: Cervical cancer; ceRNA: Competing endogenous RNA; DUXAP8: Double homeobox A pseudogene 8; EMT: Epithelial mesenchymal transition; IncRNAs: Long non-coding RNAs; nCRNAs: Non-coding RNAs; RT-qPCR: Real- time quantitative PCR; RCN: Reticulocalbin; RCN2: Reticulocalbin-2; RIP: RNA binding protein immunoprecipitation}

\section{Acknowledgements}

Members involved in are appreciated.

\section{Authors' contributions}

Jihui Gu and Wen Feng were invested a lot of time and energy in the experiments and records as well as manuscript grammar corrections; Yi Liu and Ting Qi were contributed to data curation; Weiwei Qian contributed to the investigation. Dongdong Hu supervised laboratorial processes. All authors contributed to manuscript revision, read, and approved the submitted version.

\section{Funding}

There is no funding.
Availability of data and materials

Not applicable.

\section{Declarations}

Ethics approval and consent to participate

The animal study was approved by the Ethics Committee of the First People's Hospital of Lianyungang.

Consent for publication

Not applicable.

\section{Competing interests}

The authors declare that they have no competing interests.

Received: 26 May 2021 Accepted: 30 August 2021

Published online: 14 November 2021

\section{References}

1. Wang R, Pan W, Jin L, Huang W, Li Y, Wu D, et al. Human papillomavirus vaccine against cervical cancer: opportunity and challenge. Cancer Lett. 2020;471:88-102.

2. Mapanga W, Singh E, Feresu SA, Girdler-Brown B. Treatment of pre- and confirmed cervical cancer in HIV-seropositive women from developing countries: a systematic review. Syst Rev. 2020;9(1):79.

3. Yadav N, Parveen S, Banerjee M. Potential of nano-phytochemicals in cervical cancer therapy. Clin Chim Acta. 2020;505:60-72.

4. Siegel RL, Miller KD, Fuchs HE, Jemal A. Cancer Statistics, 2021. CA Cancer J Clin. 2021;71(1):7-33.

5. Rajagopal T, Talluri S, Akshaya RL, Dunna NR. HOTAIR LncRNA: A novel oncogenic propellant in human cancer. Clin Chim Acta. 2020;503:1-18.

6. Liu B, Li J, Liu X, Zheng M, Yang Y, Lyu Q, et al. Long non-coding RNA HOXA11-AS promotes the proliferation HCC cells by epigenetically silencing DUSP5. Oncotarget. 2017:8(65):109509-21.

7. Ashrafizaveh S, Ashrafizadeh M, Zarrabi A, Husmandi K, Zabolian A, Shahinozzaman $\mathrm{M}$, et al. Long non-coding RNAs in the doxorubicin resistance of cancer cells. Cancer Lett. 2021;508:104-14.

8. Fan Y, Sheng W, Meng Y, Cao Y, Li R. LncRNA PTENP1 inhibits cervical cancer progression by suppressing miR-106b. Artif Cells Nanomed Biotechnol. 2020;48(1):393-407.

9. Yang TJ, Wang $L$, Zhang Y, Zheng JD, Liu L. LncRNA UCA1 regulates cervical cancer survival and EMT occurrence by targeting miR-155. Eur Rev Med Pharmacol Sci. 2020:24(19):9869-79.

10. Lian Y, Yang J, Lian Y, Xiao C, Hu X, Xu H. DUXAP8, a pseudogene derived IncRNA, promotes growth of pancreatic carcinoma cells by epigenetically silencing CDKN1A and KLF2. Cancer Commun (London). 2018;38(1):64.

11. Chen $P$, Zhang W, Chen $Y$, Zheng $X$, Yang D. Comprehensive analysis of aberrantly expressed long non-coding RNAs, microRNAs, and mRNAs associated with the competitive endogenous RNA network in cervical cancer. Mol Med Rep. 2020;22(1):405-15.

12. Wei F, Yang $L$, Jiang D, Pan M, Tang G, Huang $M$, et al. Long noncoding RNA DUXAP8 contributes to the progression of hepatocellular carcinoma via regulating miR-422a/PDK2 axis. Cancer Med. 2020;9(7):2480-90.

13. Du C, Wang HX, Chen P, Chen CH. STAT3-induced upregulation of IncRNA DUXAP8 functions as ceRNA for miR-577 to promote the migration and invasion in colorectal cancer through the regulation of RAB14. Eur Rev Med Pharmacol Sci. 2019;23(14):6105-18

14. Yan SP, Chu DX, Qiu HF, Xie Y, Wang CF, Zhang JY, et al. LncRNA LINC01305 silencing inhibits cell epithelial-mesenchymal transition in cervical cancer by inhibiting TNXB-mediated PI3K/Akt signalling pathway. J Cell Mol Med. 2019;23(4):2656-66.

15. Sohel MMH. Circulating microRNAs as biomarkers in cancer diagnosis. Life Sci. 2020;248:117473.

16. Tang $X$, Shi $X$, Wang N, Peng W, Cheng Z. MicroRNA-215-3p suppresses the growth, migration, and invasion of colorectal cancer by targeting FOXM1. Technol Cancer Res Treat. 2019;18:1533033819874776.

17. Nahand JS, Taghizadeh-Boroujeni S, Karimzadeh M, Borran S, Pourhanifeh MH, Moghoofei M, et al. microRNAs: New prognostic, diagnostic, and therapeutic biomarkers in cervical cancer. J Cell Physiol. 2019:234(10):17064-99. 
18. Ding $D$, Huang $H$, Jiang $W$, Yu W, Zhu H, Liu J, et al. Reticulocalbin-2 enhances hepatocellular carcinoma proliferation via modulating the EGFRERK pathway. Oncogene. 2017;36(48):6691-700.

19. Marth C, Landoni F, Mahner S, McCormack M, Gonzalez-Martin A, Colombo N. Cervical cancer: ESMO Clinical Practice Guidelines for diagnosis, treatment and follow-up. Ann Oncol. 2018;29(Suppl 4):iv262.

20. Small W Jr, Bacon MA, Bajaj A, Chuang LT, Fisher BJ, Harkenrider MM, et al. Cervical cancer: a global health crisis. Cancer. 2017;123(13):2404-12.

21. Zhu H, Luo H, Zhang W, Shen Z, Hu X, Zhu X. Molecular mechanisms of cisplatin resistance in cervical cancer. Drug Des Devel Ther. 2016;10: 1885-95.

22. Sun W, Shi Q, Zhang H, Yang K, Ke Y, Wang Y, et al. Advances in the techniques and methodologies of cancer gene therapy. Discov Med. 2019; 27(146):45-55

23. Dai X, Kaushik AC, Zhang J. The emerging role of major regulatory RNAs in cancer control. Front Oncol. 2019;9:920.

24. Ma HW, Xie M, Sun M, Chen TY, Jin RR, Ma TS, et al. The pseudogene derived long noncoding RNA DUXAP8 promotes gastric cancer cell proliferation and migration via epigenetically silencing PLEKHO1 expression. Oncotarget. 2017;8(32):52211-24.

25. Chen J, Lou W, Ding B, Wang X. Overexpressed pseudogenes, DUXAP8 and DUXAP9, promote growth of renal cell carcinoma and serve as unfavorable prognostic biomarkers. Aging. 2019;11(15):5666-88.

26. Jiang $\mathrm{H}$, Shi X, Ye G, Xu Y, Xu J, Lu J, et al. Up-regulated long non-coding RNA DUXAP8 promotes cell growth through repressing Kruppel-like factor 2 expression in human hepatocellular carcinoma. OncoTargets Ther. 2019;12: 7429-36.

27. Ashrafizadeh M, Gholami MH, Mirzaei S, Zabolian A, Haddadi A, Farahani MV, et al. Dual relationship between long non-coding RNAs and STAT3 signaling in different cancers: New insight to proliferation and metastasis. Life Sci. 2021;270:119006.

28. Mirzaei S, Zarrabi A, Hashemi F, Zabolian A, Saleki H, Ranjbar A, et al. Regulation of Nuclear Factor-KappaB (NF-KB) signaling pathway by noncoding RNAs in cancer: Inhibiting or promoting carcinogenesis? Cancer Lett. 2021:509:63-80

29. Mirzaei S, Zarrabi A, Asnaf SE, Hashemi F, Zabolian A, Hushmandi K, et al. The role of microRNA-338-3p in cancer: growth, invasion, chemoresistance, and mediators. Life Sci. 2021;268:119005.

30. Ashrafizadeh M, Zarrabi A, Hushmandi K, Zarrin V, Moghadam ER, Zabolian A, et al. PD-1/PD-L1 axis regulation in cancer therapy: the role of long noncoding RNAs and microRNAs. Life Sci. 2020;256:117899.

31. Gao C, Ren C, Liu Z, Zhang L, Tang R, Li X. GAS5, a FoxO1-actived long noncoding RNA, promotes propofol-induced oral squamous cell carcinoma apoptosis by regulating the miR-1297-GSK3beta axis. Artif Cells Nanomed Biotechnol. 2019:47(1):3985-93.

32. Chen Z, Ma Y, Pan Y, Zhu H, Yu C, Sun C. MiR-1297 suppresses pancreatic cancer cell proliferation and metastasis by targeting MTDH. Mol Cell Probes. 2018:40:19-26

33. Gao W, Cao Y, Guo P, Bao X, Zhu H, Zheng J, et al. Downregulation of MiR1297 predicts poor prognosis and enhances gastric cancer cell growth by targeting CREB1. Biomed Pharmacother. 2018;105:413-9.

34. Wang Z, He S, Guo P, Guo X, Zheng J. MicroRNA-1297 inhibits metastasis and epithelial-mesenchymal transition by targeting AEG-1 in cervical cancer. Oncol Rep. 2017;38(5):3121-9.

35. Liu Y, Mi Y, Mueller T, Kreibich S, Williams EG, Van Drogen A, et al. Multiomic measurements of heterogeneity in HeLa cells across laboratories. Nat Biotechnol. 2019;37(3):314-22.

36. Wang G, Zhou J, Lu F, Qiu L, Xu L, Yang X, et al. Downregulation of microRNA1835p inhibits the proliferation and invasion of colorectal cancer cells by inactivating the reticulocalbin2/Wnt/betacatenin signaling pathway. Mol Med Rep. 2019;19(5):4475-83.

\section{Publisher's Note}

Springer Nature remains neutral with regard to jurisdictional claims in published maps and institutional affiliations. 